\title{
Tsafon
}

Revue d'études juives du Nord

$82 \mid 2021$

Enjeux esthétiques dans la littérature après Auschwitz

\section{Lessing Theodor, Jewish-Self-Hate}

\section{Martine Benoit}

\section{(2) OpenEdition}

Journals

Édition électronique

URL : https://journals.openedition.org/tsafon/4487

DOI : 10.4000/tsafon.4487

ISSN : 2609-6420

\section{Éditeur}

Association Jean-Marie Delmaire

\section{Édition imprimée}

Date de publication : 1 décembre 2021

Pagination : 146-148

ISSN : 1149-6630

\section{Référence électronique}

Martine Benoit, «Lessing Theodor, Jewish-Self-Hate», Tsafon [En ligne], 82 | 2021, mis en ligne le 01 décembre 2021, consulté le 12 février 2022. URL : http://journals.openedition.org/tsafon/4487 ; DOI : https://doi.org/10.4000/tsafon.4487

Ce document a été généré automatiquement le 12 février 2022.

Tsafon. Revues d'études juives du Nord 


\title{
Lessing Theodor, Jewish-Self-Hate
}

\author{
Martine Benoit
}

\section{RÉFÉRENCE}

Lessing Theodor, Jewish-Self-Hate, Berghahn, New York-Oxford, 2021, traduit et annoté par Peter C. Appelbaum, introduit par Sander L. Gilman, postfacé par Paul Reitter, édité par Benton Arnovitz, 186 p.

1 Né en 1872 à Hanovre au sein d'une famille juive acculturée, Theodor Lessing était un publiciste, essayiste et professeur de philosophie, démocrate convaincu, qui s'est battu dès le tournant du siècle pour les droits des femmes et l'éducation des couches défavorisées. Républicain engagé défendant avec un courage tant moral que physique une République de Weimar malade de ses faiblesses et de ses hésitations, Juif qui a combattu l'antisémitisme en Allemagne et plaidé pour un sionisme socialiste pour venir en aide aux Ostjuden, assassiné par des hommes de main des nazis le 30 août 1933 à Marienbad où il se pensait en sécurité, il est notamment passé à la postérité par cette expression de " haine de soi juive ». Son ouvrage, Der jüdische Selbsthass, parait, aux éditions du Jüdischer Verlag, à la fin de l'année 1930, alors que l'Allemagne voit croître le nombre de députés nazis au Reichstag et, avec lui, l'antisémitisme se diffuser toujours plus profondément dans la société. C'est le dernier livre à être paru du vivant de Theodor Lessing, il est le fruit de la réflexion de toute une vie. L'avant-propos souligne: "L'auteur de ces lignes a lui aussi, dans sa jeunesse, connu une période d'adhésion exclusive à la 'germanité', de rejet absolu de la 'judéité' ». Der jüdische Selbsthass présente une série de portraits de six auteurs : Paul Rée (1849-1901), ami de Nietzsche et de Lou Andreas-Salomé ; le publiciste Maximilian Harden (1861-1927); Otto Weininger (1880-1903), auteur de Sexes et caractères (Geschlecht und Charakter) en $1903^{2}$; l'écrivain Arthur Trebitsch (1880-1927) ; le poète Walter Calé (1881-1904); Max Steiner (1884-1910), jeune scientifique qui se suicida à l'âge de vingt-six ans. Le propos central de Theodor Lessing à travers ces portraits, et les nombreuses digressions qu'il fait, est d'encourager les Juifs allemands à assumer pleinement leur judéité. 
2 En France, Maurice-Ruben Hayoun traduisait et introduisait l'ouvrage de Theodor Lessing en 1990 chez Berg International sous le titre : La haine de soi - le refus d'être juif, traduction rééditée en 2011 avec une nouvelle postface - avec ce titre, Maurice-Ruben Hayoun évitait l'obstacle du concept « haine de soi juive ». La traduction américaine, la première traduction en anglais de Der jüdische Selbsthass, a fait un autre choix : alors que jusqu'à présent, la littérature secondaire anglophone (notamment l'essai de Kurt Lewin en 1941 sous le titre "Self-hatred among Jews' »; l'article de Lawrence Baron en 1981 «Theodor Lessing: Between Jewish Self-Hatred and Zionism»; le livre de Sander L. Gilman Jewish Self-Hatred: Anti-Semitism and the Hidden Language of the Jews en 1986) utilisait pour traduire le concept de « haine de soi juive » le terme self-hatred, pour la première fois, Peter $C$. Appelbaum et son éditeur Benton Arnovitz utilisent le mot " self-hate », en effet le plus proche du concept allemand de «Selbsthass » - et ce choix est à saluer.

3 Publiée avec l'accord et les encouragements des Éditions Suhrkamp, héritières depuis 1958 des éditions du Jüdischer Verlag fermées par les nazis en 1938, cette première traduction en anglais et cette nouvelle édition est à saluer fortement. L'ouvrage de Theodor Lessing, par une traduction très appliquée et un appareil de notes très complet, devient ainsi accessible au public anglophone. Tout a été fait pour laisser à la fois la texture allemande du texte et permettre au lecteur anglophone d'aujourd'hui de lire ce texte ardu.

4 Le traducteur Peter C. Appelbaum, professeur émérite de pathologie à l'université d'État de Pennsylvanie, finalise actuellement un travail sur la place des Juifs dans l'armée austro-hongroise (à paraître : Habsburg Sons : Jews in the Austro-Hungarian Army, 1788-1918, Cherry Orchard Books). Pour sa traduction, Peter C. Appelbaum s'est entouré de deux des plus grands connaisseurs du sujet : Sander L. Gilman, désormais professeur émérite, qui publie dès 1986 son Jewish Self-Hatred : Anti-Semitism and the Hidden Language of the Jews; Paul Reitter, directeur des écoles doctorales de l'université d'État de l'Ohio, germaniste spécialiste de la culture judéo-allemande, qui a travaillé sur Karl Kraus (The Anti-Journalist : Karl Kraus and Jewish Self-Fashioning in Fin-de-Siecle Europe, University of Chicago Press, 2008) avant de consacrer en 2012 une étude à la haine de soi juive, On the Origins of Jewish Self-Hatred (aux Princeton University Press) et d'écrire l'entrée «Selbsthass » (page 553-570) dans l'Encyclopédie de l'histoire et de la culture juives, dirigée par le grand Dan Diner et parue aux Éditions Metzler en 2015 (titre original: Enzyklopädie jüdischer Geschichte und Kultur).

5 Dans sa préface de quelque 25 pages, Sander L. Gilman évoque les destins et les engagements de Leon Pinsker, Jakob Wassermann, leur positionnement vis-à-vis de l'assimilation dans un contexte où l'antisémitisme en démontrait toutes les failles et les faiblesses. Sander L. Gilman revient sur le travail d'Anna Freud autour de la notion d'identification à l'agresseur, développe, à la suite de Kurt Lewin, la notion de haine de soi chez les Noirs américains. À la fin de son avant-propos, Sander L. Gilman insiste sur le fait que « ce que nous pouvons apprendre de la trajectoire du discours de la haine de soi, c'est qu'il pourrait bien s'agir d'un phénomène universel et complexe et qu'il ne s'agit certainement pas simplement d'un phénomène propre aux Juifs acculturés » (page 35). Dans sa postface, Paul Reitter rappelle quant à lui l'accueil du livre par les sionistes : directeur des Éditions du Jüdischer Verlag, Siegmund Kaznelson y voyait un coup de propagande sioniste bienvenu. Pour Paul Reitter cependant, il y a un abîme entre la réception du livre à sa sortie et sa réception aujourd'hui, un abîme qui pose 
question. Paul Reitter s'interroge en effet sur le moment de l'apparition du concept même de " haine de soi juive » sous la plume de Theodor Lessing et estime que celui-ci n'a pu apparaître qu'après la Première Guerre mondiale. Paul Reitter développe son propos à partir de textes du journaliste autrichien d'origine juive, Anton Kuh, qui utilisa l'expression de "haine de soi juive » dans des discours des années 1918-1920, discours publiés à Berlin en 1921 aux éditions Erich Reiss sous le titre Juden und Deutsche (Juifs et Allemands) ${ }^{3}$. Selon Reitter, Anton Kuh et Theodor Lessing devaient se connaître, ils travaillaient tous les deux pour le quotidien Prager Tagblatt, journal des démocrates de langue allemande en Tchécoslovaquie. Anton Kuh aurait introduit le terme de haine de soi notamment pour son approche psychologisante et son rapport au conflit pèrefils. Le propos de Paul Reitter est de confronter les textes d'Anton Kuh au propos de Theodor Lessing.

Le texte de Theodor Lessing est, comme le souligne Peter C. Appelbaum dans son introduction, un livre sur la nature complexe de la haine de soi des Juifs allemands de la fin du XIX ${ }^{\text {ème }}$ siècle jusqu'à la République de Weimar. Peter C. Appelbaum souligne les difficultés rencontrées devant un texte sinueux, comportant de nombreuses erreurs que Peter C. Appelbaum corrige avec beaucoup de doigté par son impressionnant appareil de notes: alors que le livre de Theosor Lessing comportait 17 notes de son auteur, la traduction en comporte plus de 300. Le choix éditorial a été de placer ces notes à la fin de chaque chapitre : l'appareil de notes distingue les notes de Theodor Lessing, reproduites en italique, des notes de Peter C. Appelbaum. Placer les notes en fin de chaque chapitre permet aux lecteurs d'accéder rapidement à ces notes; cela coupe un peu peut-être la lecture du texte de Theodor Lessing. Ces notes sont cependant indispensables pour une lecture intelligente et abordable à un lecteur d'aujourd'hui : Peter C. Appelbaum y rappelle ce qu'étaient la Jewish Agency, le Bilu, la Légion juive, donne des explications sur la vie juive, explique des mots de yiddish ou d'hébreu, donne une courte présentation des grands noms que cite Lessing (de Agnon à Moses Hess, Leo Pinsker, Martin Buber, en passant par Moses Mendelssohn, Heinrich Heine, Gotthold Ephraim Lessing, Johann Wolfgang Goethe, Friedrich Schiller, Hugo von Hofmannsthal, Franz Werfel, Jakob Wassermann, Karl Kraus mais aussi Ferdinand Lassalle, Karl Marx, Friedrich Nietzsche, Arthur Schopenhauer, Sigmund Freud, Lou Andreas-Salomé, mais aussi les auteurs antisémites tels Paul de Lagarde, Julius Langbehn, Eugen Karl Dühring, Houston Stewart Chamberlain). Peter C. Appelbaum fait montre d'une profonde intégrité scientifique en n'hésitant pas à admettre dans une note qu'il n'est pas suffisamment qualifié pour expliquer tel ou tel passage (voir ainsi note 23 p. 154, sur un passage de Theodor Lessing où celui-ci confronte la pensée de Paul Rée à celle de Friedrich Nietzsche). Pour certains mots, le traducteur a préféré garder le terme allemand, choix qu'il explique en notes : ainsi des termes «Volk» ou « völkisch », « intraduisibles en anglais » (note 26 p. 155 ou 18 p. 237).

7 Cette nouvelle traduction permet de mieux comprendre aujourd'hui le texte de Lessing, souvent complexe et obscur. On ne peut donc que saluer ce double travail de traduction et d'éditorialisation - et encourager les bibliothèques universitaires françaises à faire l'acquisition de cette première traduction anglophone. 


\section{NOTES}

1. Voir à ce propos Martine Benoit, «Le phénomène de 'haine de soi juive' : de la douleur d'être Juif en Allemagne (1867-1933) ", p.149-159, Cahiers d'études germaniques 2019/02 (n77: Histoire des Juives et des Juifs d'Allemagne (1867-1933), études réunies par Laurence Guillon, Patrick Farges et Laurent Dedryvère.

2. Sur Otto Weininger voir l'excellent livre de Jacques Le Rider, Le cas Otto Weininger : racines de l'antiféminisme et de l'antisémitisme, Paris, PUF, 1982.

3. Une réédition de ce texte est parue sous la direction d'Andreas Kilcher en 2003 aux Éditions Böhlau de Vienne. Anton Kuh a été redécouvert au début des années 2000, ses œuvres complètes publiées par les Éditions Wallstein en 2016 en sept volumes sous la direction de Walter Schübler. 